\title{
Can we predict (complex) PTSD in young people in foster care?
}

\section{By Dr. Jessica K Edwards}

Many children and adolescents removed from the family home have experienced some form of maltreatment and/or trauma in their lifetime. These adverse, early life experiences put young people at risk of developing psychological difficulties. ${ }^{1}$ Potential difficulties might include post-traumatic stress disorder (PTSD) ${ }^{2}$ or the newly proposed, complex PTSD.3.4 However, there is little evidence to explain the mechanisms that might drive either PTSD or complex PTSD in this unique group of affected young people.

To address this knowledge gap, Rachel Hiller and colleagues conducted a prospective study of 120 adolescents (aged 10-18 years old) who were in out-of-home care, and their primary carers. The participants completed questionnaires regarding trauma history, PTSD symptoms and complex features. These complex features were collected using a 12-item Child Complex PTSD Checklist that assesses the three additional symptoms beyond PTSD: negative self-concept, interpersonal difficulties and affect dysregulation. ${ }^{4}$ The affected young people also gave self-reports on three cognitive processes: trauma-related maladaptive appraisals, memory quality and coping.

Hiller et al. found that the severity of maltreatment did not robustly predict PTSD or complex PTSD. The three cognitive processes, however, did moderately-to-strongly correlate with baseline and 12-month PTSD symptoms and complex features. It thus seems that the existing cognitive models of PTSD are applicable to young people who have been exposed to complex trauma - in this case young people in the care system. "The same processes that we see driving the maintenance of PTSD symptoms in other groups of young people - maladaptive cognitions, memory qualities, coping - are also applicable to young people in care", explains Hiller. "Negative or maladaptive cognitions about the meaning of their maltreatment seem to be particularly important".

Hiller suggests that these cognitive processes constitute important treatment targets for young people in care with PTSD or complex PTSD. "These processes are already targeted in our established first-line treatment for PTSD - trauma focused CBT", says Hiller. "It is now crucial that young people in care with PTSD are offered evidence-based treatments".

\section{Referring to:}

Hiller, R.M., Meiser-Stedman, R., Elliott, E., Banting, R. \& Halligan, S.L. (2020), A longitudinal study of cognitive predictors of (complex) post-traumatic stress in young people in out-of-home care. J. Child Psychol. Psychiatr. doi: 10.111/jcpp.13232.

\section{References:}

${ }^{1}$ Keyes, K.M. et al. (2012), Childhood maltreatment and the structure of common psychiatric disorders. Br. J. Psychiatr. 200: 107-115. doi: 10.1192/bjp. bp.111.093062.

${ }^{2}$ American Psychiatric Association. (2013). Diagnostic and statistical manual of mental disorders (5th edn). Arlington, VA: Author.

${ }^{3}$ Brewin, C.R. et al. (2017). A review of current evidence regarding the ICD11 proposals for diagnosing PTSD and Complex PTSD. Clin. Psychol. Rev. 58 : 1-15. doi: 10.1016/j.cpr.2017.09.001.

${ }^{4}$ World Health Organisation. (2018). International statistical classification of diseases and related health problems (11th Revision). Available from: https://icd.who. int/browse11/ I-m/en.

\section{Glossary:}

\section{Complex post-traumatic stress}

disorder: a diagnostic category introduced by the World Health Organization's classification system (ICD-11). Affected patients must meet the full criteria for PTSD, as well as exhibit (i) affect dysregulation, (ii) negative self-concept and (iii) disturbances in relationships. 